\title{
Ascending Prices and Package Bidding: A Theoretical and Experimental Analysis ${ }^{\dagger}$
}

\author{
By John H. Kagel, Yuanchuan Lien, and Paul Milgrom*
}

\begin{abstract}
We use theory and experiment to explore the performance of multiround, price-guided, combinatorial auctions. We define efficiencyrelevant and core-relevant packages and show that if bidders bid aggressively on these and losing bidders bid to their limits, then the auction leads to efficient or core allocations. We study the theoretically relevant behaviors and hypothesize that subjects will make only a few significant bids, and that certain simulations with auto-bidders will predict variations in performance across different environments. Testing the combinatorial clock auction (CCA) design, we find experimental support for these two hypotheses. We also compare the CCA to a simultaneous ascending auction. (JEL D44)
\end{abstract}

A long tradition in economic thought asserts that prices minimize the amount of communication required in arriving at an efficient allocation of resources. Friedrich A. Hayek (1945) emphasized this point as one of the keys to the success of market capitalism, and Leonid Hurwicz (1977) formalized it, utilizing the additional assumption that firms' production sets, and consumers' preferred sets, are convex. Recent theoretical results of Noam Nisan and Ilya Segal (2006) make it clear that such additional assumptions are important. In some striking examples, Liad Blumrosen and Nisan (2005) show that price-guided procedures can fail to achieve even a fraction of the maximum possible value. Worst-case theoretical analyses, however, leave open the possibility that auctions guided by individual item prices might perform well in a far wider range of environments than those studied in neoclassical economic theory.

Over the past few years, economic experimenters have explored that possibility. They have designed price-guided auction mechanisms-cousins of the Walrasian tâtonnement process - and tested them on selected allocation problems. These new

\footnotetext{
* Kagel: Economics Department, Ohio State University, 410 Arps Hall, 1945 N. High St., Columbus, OH 43210 (e-mail: kagel.4@osu.edu); Lien: Division of Humanities and Social Sciences, California Institute of Technology, Mail code 228-77, 1200 E. California Blvd., Pasadena, CA 91125 (e-mail: ylien@ylien.com); Milgrom: Economics Department, Stanford University, 579 Serra Mall, Stanford, CA 94305 (e-mail: milgrom@stanford.edu). We are grateful to the referees for their comments and suggestions. We thank Nels Christiansen and Marissa Beck who provided valuable research support; David Moshal who created the auction software; and Jo Ducey for editorial assistance. We thank Peter Cramton, Jacob Goeree, Marion Ott, and attendees at the University of Arizona conference in honor of Vernon Smith's eightieth birthday, and attendees at the Pennsylvania State University CAPCP conference for helpful comments. Kagel and Milgrom acknowledge financial help from the National Science Foundation (grant ITR-0427770), and Kagel acknowledges help from the National Science Foundation (grant SES-0648293). The experimental instructions, together with the other electronic appendices, are available on the American Economic Journal: Microeconomics Web site.

$\uparrow$ To comment on this article in the online discussion forum, or to view additional materials, visit the articles page at http://www.aeaweb.org/articles.php?doi=10.1257/mic.2.3.160.
} 
mechanisms differ from the classical Walrasian mechanism in two important ways. First, for any specified prices, the quantities bid at each round represent an actual commitment to buy the demanded bundle at the specified prices. And second, these mechanisms use both current and past bids in a single optimization to determine the winning bids, so that formerly losing bids can become winning 1

Two recent papers report pioneering experiments with such mechanisms. The RAD mechanism of Anthony M. Kwasnica et al. (2005) uses the bids at each round to compute tentative prices for each item that come "as close as possible" to market clearing prices in a particular metric. After each round, the prices are reported back to the bidders and used to set minimum bids for the next round of bidding. Individual item prices in RAD can increase or decrease from round to round. In contrast, the combinatorial clock auction (CCA) mechanism, introduced and tested by David Porter et al. (2003), determines prices for each item that increase monotonically from round to round.

Porter et al. (2003) report surprisingly efficient outcomes from an experiment testing the CCA. In 25 auction trials, efficiencies of 99 percent are reported in two trials and 100 percent are reported in the remaining 23 trials. Unfortunately, these results cannot be replicated because detailed information about the valuations used in their experiment is unavailable.

Do these experimental findings disprove the significance of the theoretical ones? Can experiments provide a way to assess "average" as opposed to "worst case" performance? Do they enable appropriate comparisons among mechanisms? The answers, we argue, are negative; only a combination of experiments and theory can be sufficient. To illustrate why, consider the experiment reported by Christoph Brunner et al. (2010), and Goeree, Holt, and Ledyard (2007), testing a RADinspired design-to-sell radio spectrum licenses for FCC auction 73. The experiment entailed selling 18 licenses: 12 "national" licenses and 6 "regional" licenses. The number of possible packages is the number of nonempty subsets of a set of 18 items, or 262,143 . So, setting aside any possible value interdependencies or externalities, the set of possible value profiles for $n$ bidders is $\Re_{+}^{262143 n}$, which is far too large to explore systematically using experimental trials alone.

In this paper, we study combinatorial auctions using a mix of theory and experiments. Our theory consists of two parts. The first asks the question, "under what conditions does a series of bids in a combinatorial auction produce allocations that are efficient or in the core?' 2 To answer that, we introduce the concepts of efficiency-relevant and core-relevant packages and of sufficiently aggressive bidding. We show that for a very broad set of auction mechanisms, if bids in the auction for the efficiency-relevant or core-relevant packages are sufficiently aggressive, then the auction outcome will be an efficient or core allocation, respectively. As the relevant

\footnotetext{
${ }^{1}$ To illustrate this important feature, suppose that three bidders are bidding for two items, $\mathrm{A}$ and $\mathrm{B}$, and that each bidder places only one bid. Bidder 1 offers 5 for item A, bidder 2 offers 5 for item B, and bidder 3 offers 11 for the bundle AB, respectively. Then, the highest total price 11 is then obtained from bidder 3's bid alone. If bidder 2 subsequently raises its bid for B to 7 , then the highest total price rises to 12 , which is obtained by combining bidder 2's new bid with bidder 1's formerly losing bid.

${ }^{2}$ The allocation of an auction is in the core if, in addition to being efficient, it is individually rational and the total price paid by each bidder and group of bidders is "competitive," meaning that it is not less than the opportunity cost of the resources the bidder or group acquires.
} 
packages are only a subset of all possible packages, this conclusion suggests that the most important difference in performance among combinatorial mechanisms may lie in their ability to elicit relevant bids.

The problem of eliciting sufficiently aggressive bids for relevant packages is made complicated by the sheer number of profitable packages in some auctions, which we hypothesize makes it likely that some packages never receive any bids. We conjecture that the bids based on price feedback are the only important ones in determining the systematic performance of auction mechanisms like RAD and CCA. This leads us to the second part of our theory - the empirical hypothesis that the performance of simulations with particular automated bidders can predict the performance of auction experiments with human subjects. The automated bidders bid myopically in each round for the currently most profitable package. Our experiment tests this hypothesis using the CCA mechanism and a closely matched version of the simultaneous ascending auction (SAA), which is a nonpackage auction that is widely used for radio spectrum sales. We use simulations to select parameters for our experiment, including some parameters predicted to lead to efficient auction outcomes ("easy" cases) and others predicted to lead to inefficient outcomes ("hard" cases) 3

Our main experimental findings are mostly consistent with our hypotheses. Subjects consistently bid on only a small number of packages in the CCA auctions. Some profitable packages never receive any bids. A large majority of bids are placed on the myopically most profitable packages, but we find that other cues can also influence the packages that subjects choose to bid on. Efficiency is significantly higher in easy than in hard cases for the CCA auctions, and the reasons line up well with the first part of our theory that the most profitable packages correspond to the relevant packages in the easy auctions, but not in the hard auctions. The easy CCA auctions have significantly higher efficiency than the corresponding SAA auctions, but there are no significant differences in efficiency between the two auction formats for those auctions classified as hard. Guided by our theory, we look closely at the auctions classified as hard and identify and characterize a subset of those auctions for which the CCA mechanism generates significantly lower efficiency than in the corresponding SAA auctions.

We also divide our test cases into "core-easy" and "core-hard cases," according to how close the simulated outcomes are to core outcomes. In contrast to the efficiency tests, the distance from the core in simulations is only weakly predictive of the same measure in the experiment. The reasons for this remain to be explored.

For the remainder of this paper: Section I presents our theoretical results, identifying sufficient conditions for obtaining efficient or core allocations in the large class of standard package auctions. Section II describes the experimental procedures. Section III presents the experimental results and tests our hypotheses. Concluding remarks are offered in Section IV.

\footnotetext{
${ }^{3}$ We do not report any distribution of selected parameters to subjects, as might be useful if we were testing Bayesian equilibrium theories based on commonly known prior beliefs. Our experiment is not designed to test such theories.
} 


\section{Theory and Hypotheses}

To facilitate comparisons of dynamic auctions with direct mechanisms, it is helpful to think of, and sometimes to refer to, the highest bid a bidder makes on a package during the course of a dynamic auction as the bidder's "reported value" for that package. If a bidder never bids for a package, it is as if the reported value is zero.

Our general theory studies two nested classes of auctions. The wider class consists of total-bid-maximizing mechanisms, which assign the goods to bidders to maximize the total reported value, that is, the total of the winning bids. An auction is standard if, in addition, it fixes bidder payments so that the allocation is in the core with respect to the reported values. Standard auctions include the menu auctions of B. Douglas Bernheim and Michael D. Whinston (1986), dynamic auctions including CCA and RAD, the ascending proxy auctions of Lawrence M. Ausubel and Milgrom (2002), the one-shot core-selecting auctions of Robert Day and Milgrom (2007) (recently adopted for use in Portuguese spectrum sales), and some auction mechanisms recently used in Ireland and the United Kingdom 4 Vickrey auctions, however, are not standard, because their outcomes can fail to be core allocations.

Our theory of standard package auctions emphasizes the role of bids placed on relevant packages, and the description of these requires introducing some notation.

Let $N$ denote the set of bidders; $G$ the set of goods on offer; $x_{j}$ the package of goods assigned to bidder $j ; v_{j}\left(x_{j}\right)$ bidder $j$ 's value for its goods; $x=\left(x_{j}\right)_{j \in N}$ the goods assignment; and $X$ the set of feasible assignments. The seller is player 0 . An allocation $(x, \tau)$ consists of a goods assignment $x$ and a vector $\tau \in \Re^{N+1}$ describing the payments by the bidders and the seller's receipts. The allocation $(x, \tau)$ is feasible if $x$ $\in X$ and $\tau_{0} \leq \sum_{j \in N} \tau_{j}$. The total value of the goods assignment $x$ is $\sum_{j \in N} v_{j}\left(x_{j}\right)$. The triple $\left(N, X,\left(v_{j}\right)_{j \in N}\right)$ defines a package allocation problem.

Associated with any package allocation problem is a cooperative game with transferable utility $(N \cup\{0\}, w)$. The players in this game are the bidders $N$ and the seller, denoted as player 0 . To define the coalitional value function, it is convenient to introduce notation associating to each coalition some value-maximizing assignment $x(S) \in \arg _{\max } \sum_{x \in X} \sum_{j \in S} v_{j}\left(x_{j}\right)$. The value of the coalition consisting of the seller and the bidders in set $S$ is $w(S)=\sum_{j \in S} v_{j}\left(x_{j}(S)\right)$. Any coalition that excludes the seller has value zero.

For any package $x_{j}$, let $\beta_{j}\left(x_{j}\right)$ denote the highest price that $j$ bids for that package during the course of the auction, with $\beta_{j}\left(x_{j}\right) \equiv 0$ if no bid is made on the package. Given such a profile of "reported values" $\beta$, we define $x^{\beta}(S) \in \arg \max _{x \in X} \sum_{j \in S} \beta_{j}\left(x_{j}\right)$ and $w^{\beta}(S)=\sum_{j \in S} \beta_{j}\left(x_{j}^{\beta}(S)\right)$. These definitions are exactly analogous to the definitions of $x(S)$ and $w(S)$ using the "actual" values $v$.

Associated with any allocation is a payoff vector or imputation $\pi$ given by $\pi_{0}$ $=\sum_{j \in N} \tau_{j}$ and, for $j \in N, \pi_{j}=v_{j}\left(x_{j}\right)-\tau_{j}$. An imputation corresponds to a feasible allocation if $\pi_{0}+\sum_{j \in N} \pi_{j} \leq w(N)$. A feasible allocation $(x, \tau)$ is a core allocation and the corresponding imputation $\pi$ is a core imputation if $\pi$ is individually rational

\footnotetext{
${ }^{4}$ Formally, the simultaneous ascending auction (SAA), without explicit package bids, is another total-bid maximizing auction if one specifies that the bid for any package at any round is the sum of the individual bid prices on the lots included in the package. Theorem 2 can then be usefully applied to the SAA as well.
} 
$\left(\pi_{i} \geq 0\right.$ for $\left.i \in N \cup\{0\}\right)$ and satisfies the no-blocking inequalities $\pi_{0}+\sum_{j \in S} \pi_{j} \geq$ $w(S)$ for every set of bidders $S$. We denote by $\operatorname{Core}(N, X, v)$ the set of all core allocations for the package allocation problem $\left(N, X,\left(v_{j}\right)_{j \in N}\right)$.

We can similarly define the reported core allocations and imputations by replacing $w$ by $w^{\beta}$ and using reported profits $\pi_{j}^{\beta}=\beta_{j}\left(x_{j}\right)-\tau_{j}$. An auction is total-bid-maximizing if for every profile of bids $\beta$, it selects an assignment $x \in \arg _{\max } \sum_{z \in X} \sum_{j \in N} \beta_{j}$ $\left(z_{j}\right)$. It is standard if, in addition, for every profile of reported values, it selects a reported core allocation $(x, \tau) \in \operatorname{Core}(N, X, \beta)$.

We say that a set of bidders $S$ is core-relevant if there is some core imputation $\pi$, such that $\pi_{0}+\sum_{i \in S} \pi_{i}=w(S)$. Then, for each $j \in S$, the package $x_{j}(S)$ is bidder $j$ 's core-relevant package. Similarly, the package $x_{j}(N)$ is $j$ 's efficiency-relevant package. By a standard property of systems of linear inequalities, the core imputations are those satisfying $\pi_{0}+\sum_{j \in S} \pi_{j} \geq w(S)$ for every core-relevant set of bidders $S$. According to the next two theorems, it is sufficiently aggressive bidding (as defined by a certain inequality) on relevant packages that leads an auction to result in core allocations or efficient allocations.

THEOREM 1: In a standard package auction, suppose the outcome $(\bar{x}, \tau)$ is individually rational and let $\beta$ denote the final bids in the auction. If for all core-relevant sets of bidders $S$ and for all $j \in S, v_{j}\left(x_{j}(S)\right)-\beta_{j}\left(x_{j}(S)\right) \leq v_{j}\left(\bar{x}_{j}\right)-\beta_{j}\left(\bar{x}_{j}\right)$, then $(\bar{x}, \tau) \in \operatorname{Core}(N, X, v)$.

\section{PROOF 1:}

Since individual rationality is given, we need only establish the feasibility and the no-blocking inequalities.

For feasibility, observe that $w(N) \geq \sum_{j \in N} v_{j}\left(\bar{x}_{j}\right)=\pi_{0}+\sum_{j \in N} \pi_{j}$. The first inequality follows from the definition of $w(N)$, the second from the definition of the imputations.

For no-blocking, let $S$ be a relevant set of bidders. In a standard auction:

$$
\begin{aligned}
\pi_{0}^{\beta}+\sum_{j \in S} \pi_{j}^{\beta} & \geq w^{\beta}(S) \\
& =\max _{z \in X} \sum_{j \in S} \beta_{j}\left(z_{j}\right) \\
& \geq \sum_{j \in S} \beta_{j}\left(x_{j}(S)\right) \\
& \geq \sum_{j \in S}\left(v_{j}\left(x_{j}(S)\right)-v_{j}\left(\left(\bar{x}_{j}\right)\right)+\beta_{j}\left(\bar{x}_{j}\right)\right) \\
& =w(S)-\sum_{j \in S}\left(v_{j}\left(\bar{x}_{j}\right)-\tau_{j}\right)+\sum_{j \in S}\left(\beta_{j}\left(\bar{x}_{j}\right)-\tau_{j}\right) \\
& =w(S)-\sum_{j \in S}\left(\pi_{j}-\pi_{j}^{\beta}\right) .
\end{aligned}
$$

The first inequality holds because the auction is standard and selects an allocation $(\bar{x}, \tau) \in \operatorname{Core}(N, X, \beta)$. The second line is the definition of $w^{\beta}$ and the third follows 
from maximization. The fourth follows from the hypothesis of the theorem and the last two lines merely rearrange terms and apply definitions. Comparing the first and last terms and recalling that $\pi_{0}^{\beta}=\sum_{j \in N} \tau_{j}=\pi_{0}$, we have $w(S) \leq \pi_{0}+\sum_{j \in S} \pi_{j}$, so the coalitional no-blocking constraints are satisfied.

THEOREM 2: In a total-bid maximizing package auction, let $\beta$ denote the final bids in the auction and $(\bar{x}, \tau)$ the auction outcome. If for all bidders $j, v_{j}\left(x_{j}(N)\right)-$ $\beta_{j}\left(x_{j}(N)\right) \leq v_{j}\left(\bar{x}_{j}\right)-\beta_{j}\left(\bar{x}_{j}\right)$, then the goods assignment $\bar{x}$ is efficient: $\sum_{j \in N} v_{j}\left(\bar{x}_{j}\right)$ $=w(N)$. If the efficient goods assignment is unique, then the condition $v_{j}\left(x_{j}(N)\right)-$ $\beta_{j}\left(x_{j}(N)\right) \leq v_{j}\left(\bar{x}_{j}\right)-\beta_{j}\left(\bar{x}_{j}\right)$ is necessary as well as sufficient for $\bar{x}$ to be efficient.

\section{PROOF 2:}

We calculate as follows:

$$
\begin{aligned}
\sum_{j \in N} v_{j}\left(\bar{x}_{j}\right) & =\sum_{j \in N} \beta_{j}\left(\bar{x}_{j}\right)+\sum_{j \in N}\left(v_{j}\left(\bar{x}_{j}\right)-\beta_{j}\left(\bar{x}_{j}\right)\right) \\
& \geq \sum_{j \in N} \beta_{j}\left(x_{j}(N)\right)+\sum_{j \in N}\left(v_{j}\left(\bar{x}_{j}\right)-\beta_{j}\left(\bar{x}_{j}\right)\right) \\
& \geq \sum_{j \in N} v_{j}\left(x_{j}(N)\right)=w(N) .
\end{aligned}
$$

The first equality is an identity. The first inequality is justified by the definition of a total-bid-maximizing package auction. The second inequality follows from the hypothesis of the theorem and the final equality follows from the definition of $x_{j}(N)$. That proves the first assertion.

For the second, suppose that $\bar{x}$ is efficient. Since the efficient goods assignment is assumed to be unique, $\bar{x}=x(N)$, so $v_{i}\left(x_{i}(N)\right)-\beta_{i}\left(x_{i}(N)\right)=v_{i}\left(\bar{x}_{i}\right)-\beta_{i}\left(\bar{x}_{i}\right)$.

Informally, the theorems say that if bidders bid sufficiently aggressively for their efficiency-relevant or core-relevant packages in some particular auction, then the outcome is efficient or in the core, respectively. The conditions in these theorems are implied by equilibrium conditions in certain complete information auction mechanisms (Bernheim and Whinston 1986; Ausubel and Milgrom 2002; Day and Milgrom 2007). In the selected Nash equilibria of these earlier papers, bidders bid equally aggressively on all packages, so the conditions of Theorem 1 are satisfied and the equilibrium outcomes are core allocations.

As previously observed, the sheer number of possible packages in a large auction ensures that a bidder can bid on only a subset of its profitable packages. What might guide bidders in such settings to bid on their relevant packages, as the theorem requires? In experiments, various cues could influence bids. A subject might bid for a regional package of spectrum licenses because that package appears to be most profitable at the quoted prices, or because the bidder's role in the experiment is labeled "regional bidder," or because the experiment assigns the bidder low or zero values for licenses outside the named region, or because the bidder enjoys value 
synergies only among licenses in that region, etc. Yet such cues may be weak and are equally available to bidders in different combinatorial auction mechanisms.

We conjecture that the CCA and similar mechanisms, ones in which the information feedback in each round consists of information about winning bids and proposed prices, can perform consistently well in a class of environments only if bidder strategies using only that information can identify the relevant packages in those environments. To formalize that conjecture, we simulate auction results using automated bidders programmed to bid at each round only for the most profitable bundle at the prevailing prices. In some settings, the simulation does lead reliably to efficient or core allocations, but in others, it does not. Our conjecture is then that variations mechanism performance across different environments will be well predicted by the simulation results.

To summarize, our proposed analysis of package bidding thus entails two central hypotheses:

- In price-guided package auctions, at each round, bidders bid for many fewer packages than are profitable at the current round prices. When there are many items for sale, it is typically impossible for bidders to bid on even a small fraction of the full set of packages. But even with fewer items, subjects may be unwilling to spend the effort to bid for many packages. And, it may not serve the bidder's interest to bid for multiple packages, because that may drive up prices for other preferred bundles. In our design, bidders with provisionally winning bids may refrain from bidding at higher prices in the hopes of winning at a low price. Without specifying any single reason, our hypothesis here is that bidders bid for only one or a few packages even in small-scale auctions like those in our experiment.

- Simulations in which automated bidders bid only for the currently most profitable package will lead to (near) core or efficient outcomes in the same environments where experimental outcomes lead to approximate core or efficient outcomes. This hypothesis is based on the idea that much of what subjects do during an auction experiment is irrelevant. The auction outcome is guided mainly by bidders responding myopically in each round to price signals and bidding for the currently most profitable package.

For our experiment, we have identified two features that could subtly affect the experimental findings. First, in most of the cases we examine, the bidders have only one core-relevant package, which makes it much easier for their bids to satisfy the conditions of Theorem 1. Second, in the version of the CCA that we investigate, bidders are informed when they have a provisionally winning bid. That has turned out to be a consequential decision. The provisionally winning bidder status may become a trap if the bidder, perhaps hoping to get a low price, declines to increase its bid while it remains a provisional winner. By the time its status changes and its bid is no longer a provisional winner, prices may have risen too high to make it profitable to bid again. In terms of the theorems, we can describe these situations as failures of bidders to bid sufficiently aggressively for the relevant packages. 
North Regional

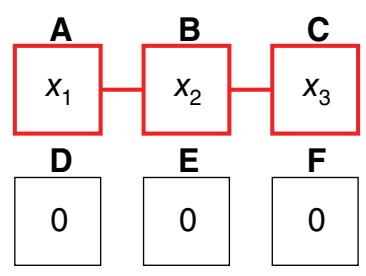

Synergies between:

- 5 and 15 or

- 25 and 35

Stand-alone values between

5 and 75 on $A \& B$ \& $C$, or D

\& $E$ \& $F$
South Regional

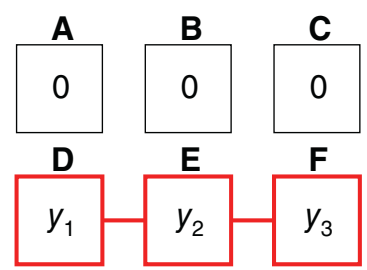

\section{Global}

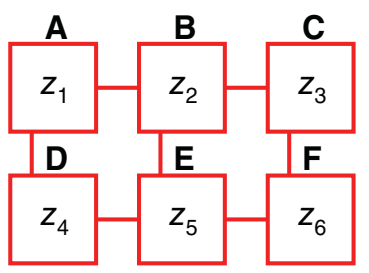

Synergies between 25 and 35

Stand-alone values between $\mathbf{5}$ and

45 on all items

Figure 1. VAluations For SiX-Item EXPERIMENT

In addition to reporting statistics about these two hypotheses, we also report on certain aspects of bidder behavior and we compare the performance of the CCA to a nonpackage auction alternative, the SAA auction. For bidder behavior, we ask, 'In our package design, do the provisionally winning bidders stand pat or make new bids?' 'In the nonpackage design, when there is an exposure problem, how do bidders respond?' 'Do they withdraw, avoiding losses but possibly missing out on potential profits?' 'Or do they continue to bid and risk suffering losses?' For evaluating performance, we analyze the auctions in terms of efficiency, revenue, bidder profits, distance to the core, and number of rounds to completion.

Before summarizing our findings, we describe the details of the experimental design.

\section{Experimental Design and Procedures 5}

We conducted auctions for either four or six items. Since we use similar value structures in both cases, we give a detailed description only for the six-item case, as illustrated in Figure 1.

There were three bidders in each auction. The north "regional bidder" had a positive value only for items $\mathrm{A}, \mathrm{B}$, and $\mathrm{C}$, and earned a positive synergy value in case it acquired two adjacent items, either $\mathrm{A}$ and $\mathrm{B}$ (henceforth $\mathrm{AB}$ ) or $\mathrm{B}$ and $\mathrm{C}$ (henceforth $\mathrm{BC}$ ). If the bidder acquired $\mathrm{ABC}$, it enjoyed two synergy values. Similarly, there was a south regional bidder with positive value for items D, E, and F, with zero value for the other items, and with identical, positive synergies between items

\footnotetext{
5 The full set of instructions, which includes sample screen shots, is available at http://www.econ. ohio-state.edu/kagel/KLM_instructions.pdf.
} 
$\mathrm{D}$ and $\mathrm{E}$ and items $\mathrm{E}$ and F. Finally, there was a "global bidder" with positive value for all six items and identical synergies for all adjacent pairs: $\mathrm{AB}, \mathrm{BC}, \mathrm{DE}, \mathrm{EF}$, $\mathrm{AD}, \mathrm{BE}$, and $\mathrm{CF}$.

For our simulations and the experiment, the stand-alone values for the regional bidders were integers from the interval $[5,75]$. There was a single synergy value for each bidder between any pair of adjacent items, which was an integer from $[5,15]$ in the low synergy regime or from $[25,35]$ in the high synergy regime. The high- or low-synergy regime was in place for both regional bidders at the same time and was announced prior to each auction. The stand-alone values for global bidders were integer values from $[5,45]$ and synergy values were integers from $[25,35]$. An online data file records the actual value profiles used in our experiment.

The four-item auctions were the same as the six-item auctions, but with standalone items $\mathrm{C}$ and $\mathrm{F}$ dropped. In both cases bidders knew the auction structure-as they were provided with a copy of Figure 1, as well as a detailed description of the possible synergy relationships and stand-alone values. However, in any particular auction they got to see only their own valuations.

After the simulations, we selected valuations for the experiment that we deemed useful for testing hypothesis 2 . We chose some cases in which repeated simulations of the CCA were 100 percent efficient ("easy" cases) and others in which they were not ("hard" cases) 6 We then selected certain easy and hard cases for the four-item and six-item treatments (see Table 5 below for the number of valuations in each category).

During the analysis phase to follow, we also make some additional distinctions. We distinguish between medium-hard and very-hard cases depending on the availability of nonprice cues that may help bidders to identify their efficiency-relevant packages - a distinction that our theory suggests could influence the efficiency of outcomes. And, in analyzing distances from the core, we distinguish cases according to how close the simulation outcomes are to core allocations.

\section{A. CCA Auctions}

Our auctions were run using a variant of the CCA rules of Porter et al. (2003). Subjects could bid on one or more packages, such as the package ABC (containing A, B and C). Bids for each bidder were XOR bids, meaning that only one of the bids could be a provisionally winning bid in any given round of the auction. In the CCA, when a bid won, the bidder was assigned all the items in its winning package and only those items.

In each round, bidders observed the prices for each item and decided about which packages to bid on. Each resulting package bid is a pair consisting of the named package and a single package price equal to the sum of the current round prices of the included items. Subjects could submit as many package bids as they wished. At the end of each round, tentative winning bids were determined from among all current and past bids by finding the feasible combination that maximized seller

\footnotetext{
${ }^{6}$ Repeated simulations may lead to different results because ties for the high bid are resolved randomly.
} 
revenue 7 Prices associated with past bids were based on prices in the round in which the bids were originally placed.

Prices for all items started at 5 ECUs (experimental currency units), and prices were raised according to the following rules. Look at the set of provisionally winning bids in the previous round and the set of new bids in the current round. If an item attracts a new bid from two or more bidders, or if it is named in a provisionally winning bid and a new bid, then its price is raised by 5 ECUs. Otherwise, the item price remains the same ${ }^{8}$ Thus, by looking at which items had price increases for the current round, bidders could easily identify items for which others were actively competing.

Following each round, bidders were privately informed about which, if any, of their bids was provisionally winning. 9

Subjects were encouraged to place bids on multiple potentially profitable packages, particularly early on as “ ... the opportunity to make profitable bids on individual items or packages with low synergies, which may become provisional winners later in the auction, will only be present early in the auction.' 10 There were no activity rules restricting the items subjects could bid on.

An auction ended after two consecutive rounds of no new bids or, what amounts to the same thing, no price increases. Two rounds were used to give everyone a chance to determine whether they were satisfied, given current prices, with their provisionally winning allocations.

\section{B. SAA Auctions}

Our SAA screen was designed to look the same as the CCA screen, so that differences in comparative performance could not be attributed to differences in presentation. The rules were also designed to be as similar as possible. Like the CCA, the SAA proceeded in a series of 25 -second rounds. And, like the CCA, a subject only had to click "set" next to any set of items to place a bid on those items. However, unlike the CCA, an SAA bidder could only make one such bid and that bid was interpreted and processed as a collection of independent item bids rather than as a package bid.

In each round, for each item with excess demand, the price was increased by 5 ECUs. The auction ended once there was no longer excess demand for any item, and each item was sold at the current price. Thus, a bidder who bid more than his or her stand-alone value for an individual item, in order to capture the synergy payoff, was exposed to a possible loss from winning just one item and paying more than its standalone value. Our version of the SAA had a number of rules and features not present in the CCA.

\footnotetext{
${ }^{7}$ Ties for tentative winning bids, which are to be expected early on in the auction, were broken randomly with priority given to tentative winners in the previous round if prices did not change. Ties become less of a concern in later stages of the auction.

${ }^{8}$ Prices were thus weakly increasing from round to round, unlike RAD (Kwasnica et al. 2005) or the FCC's Modified Package Bidding.

${ }^{9}$ Tentative winning bids were not announced in either the original Porter et al. (2003) experiment or in Brunner et al. (2007).

${ }^{10}$ In a mechanism design experiment, the instructions are an important part of the treatment as bidders are informed of the favorable properties and operation of what will typically be a novel institution.
} 
Activity Requirement.-Each auction started with bidders eligible to bid on all items - six in this case. In subsequent rounds, the total number of items a bidder was eligible to bid on could not exceed the number bid on in the previous round. This activity rule, which resembles the rule used in spectrum auctions, was explained to bidders as necessary to have the auction close in a timely manner.

Default Bids.-Each round of the auction started with a default bid labeled "currently demanded bid" which was the previous round's bid (or a bid on all items in the first round of bidding). Any time a new bid was entered that reduced eligibility, the bidder was notified and required to reconfirm the bid 11

Minimum Bid Requirement.-Once there was no longer any excess demand for an item, the current high bidder could not withdraw the bid for that item, with this requirement in effect until someone else topped that bid. This minimum bid requirement held regardless of whether there was a positive profit on the item (or set of items) in question.

Price Rollback Rule.-Given the indivisibilities inherent in the fixed-price increase rule, near the end of an auction it would not be unusual for two bidders to drop their demand for the same item at the same time, moving from excess demand to zero demand. This could result in unsold items with a potentially large, negative impact on efficiency. A price rollback rule, described in detail in the experimental instructions, was designed to deal with this situation.12 This rule randomly assigned the item in question to one of the bidders demanding the item in the previous round, at the previous round's price.

\section{Computer Interface and Aids for Subjects}

Auctions with multiple items and synergies among them are quite complicated for subjects, so the nature of the bidder interface and any analytic tools it includes can affect bidder behavior, and hence, experimental outcomes. Since we intended the experiment to be representative of high-quality field implementations, we aimed to have as friendly a computer interface as possible, as well as to provide subjects with computational aids they might expect to have from support staff in a field setting. These aids consisted of a table listing all possible bids, with corresponding analytic information, so that subjects could bid on items by simply clicking on the "add" or "set" space next to packages they were interested in. To make it easy for bidders to compare alternative packages, the table could be sorted using a number of potentially relevant criteria (e.g., current cost, current profit, etc.). 13 A

\footnotetext{
${ }^{11}$ An earlier set of SAA auctions showed that without these proactive procedures a number of subjects let their eligibility lapse well before it was profitable to do so. See the online Appendix at http://www.econ.ohio-state. edu/kagel/KLM2010AppendixOnline.pdf for a comparison of outcomes in these earlier SAA auctions with the ones reported on here.

${ }^{12}$ The minimum bid requirement would not apply in this case, as there would be no current high bidder for the item in question.

${ }^{13}$ See the online instructions for complete details regarding this and the rest of the bidder aids provided.
} 
double-criterion sort routine was employed so that a bidder interested in comparing a particular group of bids could do so easily. In the case of the six-item auctions, this was adapted so that the sort routines for regional bidders first sorted based on packages containing only those items with positive values, followed by all remaining packages ${ }^{14}$ The same set of aids was provided for the SAA and CCA auctions.

\section{Experimental Procedures}

Subjects were recruited to participate in a series of three sessions taking place within a two-week period, with each session lasting for approximately two hours. Within each series, all of the auctions had the same auction mechanism-SAA or CCA — and the same number of items (four or six). The first meeting was a training session where subjects were introduced to the experimental procedures and computer interface, followed by three dry runs, which were all that could be completed in the initial two-hour period. To insure a high return rate, subjects were offered a $\$ 30$ participation fee, to be paid only after the completion of all three sessions, and half of session 2's profits from the auctions were similarly withheld until the completion of all three sessions. In addition, subjects were paid a flat $\$ 10$ at the end of the initial training session in lieu of any earnings from the dry runs. Given the complicated nature of the auctions, subjects were permitted to take the instructions home. Earnings in sessions 2 and 3 were advertised to range between $\$ 10$ and $\$ 60$ or more per person, with average earnings of $\$ 30-\$ 40$ per person. Payoffs were denominated in experimental currency units (ECUs), with a minimum conversion rate of $1 \mathrm{ECU}=\$ 0.20 .15$ Subjects were provided with starting capital balances of 150 ECUs. Any profits earned in an auction were added to these starting capital balances, and losses subtracted from it, with total earnings for a session consisting of a subject's end-of-session balance, less 130 ECUs, but not less than zero.

Subjects' roles as a regional or global bidder were randomly determined prior to each auction, with bidders in each auction group randomly rematched following each auction. Each experimental session was designed to have five or more auctions (all with the same valuations) running at the same time. In case the number of subjects was not a multiple of three, the extras became observers for that auction, and were guaranteed to be active in the next auction. Subjects' computer screens reported only their own outcome until the end of the auction, when the full allocation of units to all bidders in their auction was reported along with a final analytics screen that they could play with. The latter was designed to give bidders a chance to see what profitable packages (according to the ending prices) they might have missed bidding on.

Each auction began with a notification to bidders about their valuations. Bidders were then given a couple of minutes to sort packages and to check any items/packages they might be particularly interested in. The six-item auctions started out

\footnotetext{
${ }^{14}$ Automatic check marks for regional bidders were only employed in the six-item auctions. These were initiated on account of the increased number of packages available to bid on. In the four-item auctions, bidders could effectively see all the packages on a single screen so the risk of mistakenly choosing dominated packages was not as severe.

${ }^{15}$ In some sessions, the total subject earnings at the minimum conversion rate was very low, so we adjusted the conversion rate upward after completion of the session.
} 
Table 1 -Experimental Treatments

\begin{tabular}{lccc}
\hline \hline Session & Session $1^{\mathrm{b}}$ & $\begin{array}{c}\text { Number of subjects }^{\mathrm{a}} \\
\text { Session 2 }\end{array}$ & Session 3 \\
\hline $\begin{array}{l}\text { Combinatorial clock auction }(C C A) \\
4 \text { items }\end{array}$ & 22 & 20 & 18 \\
& $(3)$ & $(9)$ & $(8)$ \\
6 items & 19 & 18 & 16 \\
& $(3)$ & $(9)$ & $(10)$ \\
\hline Simultaneous ascending auction $($ SAA) & & 19 \\
4 items & 21 & 20 & $(8)$ \\
6 items & $(3)$ & $(9)$ & 19 \\
& 21 & 21 & $(10)$ \\
\hline
\end{tabular}

\footnotetext{
${ }^{a}$ Number of auction valuations in each session in parentheses. Same subjects participated in a given series (e.g., CCA with four items). Number of subjects decreases over sessions due to attrition.

${ }^{\mathrm{b}}$ Data from Session 1, the training sessions, are not included in the analysis.
}

with each auction round lasting 25 seconds. After round 6 or 7, the round time was reduced to 20 seconds, and it was reduced further to 15 seconds after round 12 or so, to speed things up. Once these shorter round times went into effect, the auctioneer announced "round ending" a second or two prior to the round actually ending 16

Table 1 lists the auction sessions conducted, along with the number of subjects and the number of different valuations employed in each session ${ }^{17}$ Subjects were recruited through e-mail lists of students taking economics classes at Ohio State University in academic year 2006-2007. For subjects completing all three sessions, average earnings per subject for the six-item auctions were $\$ 174$, with minimum earnings of $\$ 90$ and maximum earnings of $\$ 331$, including the $\$ 30$ show-up fee and the $\$ 10$ payment for the first session. Average earnings per subject for the fouritem auctions were $\$ 125$, with minimum earnings of $\$ 51$ and maximum earnings of $\$ 243$, including the $\$ 30$ show-up fee and the $\$ 10$ payment for the first session.

\section{Experimental Results}

By design, our analysis is organized into easy and hard groups of parameters. In our CCA simulations, bidders bid on the most profitable packages equally in the easy and hard cases, so it will be interesting to test whether experimental subjects

\footnotetext{
${ }^{16}$ Four-item auctions, which were conducted first, had fixed round times of 25 seconds. The procedure was changed in anticipation of a larger number of rounds in the six-item auctions. Bidders appeared to have no trouble keeping up with this pace of rounds; they were canvassed regarding whether or not they had enough time to bid on all the packages they wanted to both during and after the pilot sessions. Some even complained that the pace was too slow.

${ }^{17}$ There were two sets of pilot experiments, which are not reported for both CCA and SAA auctions. They were used to refine the auction mechanisms so they would run smoothly and quickly, as well as our experimental procedures (e.g., would it really take most of two hours to go over the software and run a handful of auctions?). The number of valuations employed in each session was determined in advance. The same set of valuations was employed in the same sequence between corresponding CCA and SAA auctions. The number of auctions was not announced in advance.
} 
Table 2-Packages Bid on in CCA Auctions ${ }^{\mathrm{a}}$

\begin{tabular}{|c|c|c|c|c|c|c|}
\hline & \multicolumn{3}{|c|}{ Global bidders } & \multicolumn{3}{|c|}{ Regional bidders ${ }^{b}$} \\
\hline & \multirow[b]{2}{*}{$\begin{array}{l}\text { Average } \\
\text { number of } \\
\text { bids }^{c}\end{array}$} & \multicolumn{2}{|c|}{ Distribution of bids ${ }^{\mathrm{d}}$} & \multirow[b]{2}{*}{$\begin{array}{l}\text { Average } \\
\text { number of } \\
\text { bids }^{c}\end{array}$} & \multicolumn{2}{|c|}{ Distribution of bids ${ }^{\mathrm{d}}$} \\
\hline & & $\begin{array}{c}\text { Percent } \\
\text { most } \\
\text { profitable }\end{array}$ & $\begin{array}{c}\text { Percent } \\
\text { 2nd most } \\
\text { profitable }\end{array}$ & & $\begin{array}{c}\text { Percent } \\
\text { most } \\
\text { profitable }\end{array}$ & $\begin{array}{c}\text { Percent } \\
\text { 2nd most } \\
\text { profitable }\end{array}$ \\
\hline \multicolumn{7}{|l|}{$\overline{\text { CCA4 Auctions }}$} \\
\hline Rounds 1-5 & $\begin{array}{c}3.9 \\
(13.8)\end{array}$ & 76.7 & 43.9 & $\begin{array}{l}1.5 \\
(2.8)\end{array}$ & 91.0 & 44.4 \\
\hline Rounds 6-10 & $\begin{array}{c}1.6 \\
(7.4)\end{array}$ & 81.7 & 16.6 & $\begin{array}{c}1.2 \\
(2.3)\end{array}$ & 84.7 & 35.3 \\
\hline Rounds $11-15$ & $\begin{array}{c}1.3 \\
(4.2)\end{array}$ & 86.6 & 19.4 & $\begin{array}{c}1.1 \\
(2.1)\end{array}$ & 87.5 & 26.3 \\
\hline Rounds $>15$ & $\begin{array}{c}1.2 \\
(3.6) \\
\end{array}$ & 87.5 & 12.5 & $\begin{array}{c}1.1 \\
(1.8)\end{array}$ & 89.1 & 15.5 \\
\hline \multicolumn{7}{|l|}{$\overline{\text { CCA6 Auctions }}$} \\
\hline Rounds $1-5$ & $\begin{array}{c}11.5 \\
(60.5)\end{array}$ & 79.0 & 55.4 & $\begin{array}{c}3.1 \\
(6.8)\end{array}$ & 81.1 & 54.6 \\
\hline Rounds 6-10 & $\begin{array}{c}2.6 \\
(35.7)\end{array}$ & 72.9 & 32.4 & $\begin{array}{c}1.9 \\
(5.5)\end{array}$ & 77.7 & 45.6 \\
\hline Rounds $11-15$ & $\begin{array}{c}2.0 \\
(13.3)\end{array}$ & 85.2 & 27.2 & $\begin{array}{c}1.5 \\
(3.9)\end{array}$ & 78.6 & 40.2 \\
\hline Rounds $>15$ & $\begin{array}{c}1.3 \\
(6.9)\end{array}$ & 86.8 & 13.9 & $\begin{array}{c}1.3 \\
(3.2)\end{array}$ & 93.1 & 26.3 \\
\hline
\end{tabular}

\footnotetext{
${ }^{a}$ Data are only included for rounds in which a bidder is not a provisional winner, had at least one profitable package to bid on, and a bid was submitted.

${ }^{\mathrm{b}}$ Only includes packages where all items had positive value for regional bidders.

${ }^{\mathrm{c}}$ In parentheses are average number of profitable packages available to bid on.

${ }^{\mathrm{d}}$ Percentages add up to more than 100 percent as subjects bid on the most profitable package as well as the second most profitable package.
}

behave in the same way. We begin by showing that they do indeed. We then pool data from the two cases to report the characteristics of individual bidder behavior that our theory suggests can be consequential for auction outcomes. Finally, we compare the performance of the two auction mechanisms in terms of efficiency, seller revenues, bidder profits, distance from the core, and rounds to completion.

\section{A. Patterns of Individual Bidding}

Subjects' bidding behavior in the CCA auctions exhibits a number of consistent characteristics that our theory identifies as consequential.

First, as hypothesized, bidders bid on only a small number of profitable packages, with the most profitable package attracting the most attention. Further, these patterns do not differ materially between hard versus easy auctions. This is potentially important, because if subjects bid on more packages in one of the cases, that would make it more likely that they bid on any particular relevant package in that case.

Table 2 summarizes these data. Columns 2 and 5 of Table 2 report the average number of packages bid on in each round, along with the number of profitable packages available to bid on (in parentheses) for global and regional bidders, respectively. The columns following these show where the bids were directed in terms of the percentage of times subjects bid on the most profitable and second-most 
profitable packages, respectively 18 Data are excluded for the last two rounds of each auction where by definition there are no new bids as well as rounds in which the bidder is a provisional winner (which will be covered in detail below). So, for example, in rounds $1-5$ in the CCA4 auctions, global players bid on 3.9 packages per round on average (out of 13.8 profitable packages available to bid on), of which 76.7 percent were directed at the most profitable package.

As predicted, players bid on only a small number of the profitable packages at each round and omitted bidding on others. They did so even in later rounds when there were relatively few profitable packages-e.g., in rounds 11-15 in the CCA6 auctions. global bidders bid on only 2.0 out of 13.3 profitable packages available to bid on 19 Further, we are unable to reject a null hypothesis in any of the cells that the number of packages bid on is the same between easy versus hard auctions. Nor can we reject a null hypothesis that subjects direct their bids to the most profitable and second most profitable packages with equal frequencies in hard versus easy cases 20

To summarize, players bid on only a small percentage of the profitable packages in each round and omit some packages entirely from their bidding during the auction; bids are largely directed at the most profitable packages; and we are unable to reject a null hypothesis that the number of packages bid on, and the frequency of bidding on the most profitable package, are the same between hard versus easy auctions.

If CCA prices fail to guide effective bidding, and bidders bid on only few packages in each round, our theoretical conditions could still be satisfied if bidders bid sufficiently aggressively on all of their packages at appropriate times during the auction. But this is far from what we observe. A global bidder on average bids at least once on only 6.0 distinct packages out of the 15 packages they could bid on during the course of a CCA 4 auction. In other words, on average 9.0 packages never receive any bid at all from the global bidder during the auction (and hence correspond to a reported value of zero). Regional bidders come closer to the necessary requirement: on average, they bid at least once during the auction on 2.1 distinct packages out of 3 packages. For CCA6, the averages are 17.6 out of 63 packages for the global bidders and 5.1 out of 7 packages for the regional bidders.

Second, most losing bidders in the auction had fully exhausted their profit opportunities on their selected packages by the last bidding round. This behavior is part of the sufficient conditions for both Theorems 1 and 2.

Table 3 reports the scope for potential profits available at the end of the auction, distinguishing between losing and winning bidders. The most notable element here is the difference between regional and global bidders in the frequency with which losing bidders could have possibly obtained higher positive profits by continuing to bid, averaging 6.7 percent for global bidders versus 25.0 percent for regional

\footnotetext{
${ }^{18}$ These percentages are independent of each other in that a bid on the second most profitable package is counted regardless of whether or not a bid was placed on the most profitable package.

${ }^{19}$ In repeated canvassing, the subjects indicated that they had enough time to bid on all the packages they wanted, so this finding is not driven by time limits in the auction.

${ }^{20}$ These statistical tests involved two-tailed $(p=0.05)$ nonparametric Wilcoxon signed-rank tests with average subject data as the unit of observation. The data corresponding to Table 2 broken out by hard versus easy cases is reported in the online Appendix http://www.econ.ohio-state.edu/kagel/KLM2010AppendixOnline.pdf.
} 
TABle 3-Scope for Increased Profit at End of Auctions

\begin{tabular}{|c|c|c|c|}
\hline & Bidder type & $\begin{array}{c}\text { Frequency higher } \\
\text { profits were } \\
\text { available }{ }^{\mathrm{a}} \\
\text { (percent) }\end{array}$ & $\begin{array}{c}\text { Average foregone } \\
\text { (potential) profits in } \\
\text { ECUs }^{\mathrm{b}}\end{array}$ \\
\hline \multirow[t]{2}{*}{$\begin{array}{l}\text { CCA4 Auctions } \\
\text { Losing bidders }\end{array}$} & Global & $\begin{array}{c}4.5 \\
(3 / 66)\end{array}$ & $\begin{array}{l}12.0 \\
(7.6)\end{array}$ \\
\hline & Regional & $\begin{array}{c}25.5 \\
(12 / 47)\end{array}$ & $\begin{array}{c}14.9 \\
(15.6)\end{array}$ \\
\hline \multirow[t]{2}{*}{ Winning bidders } & Global & $\begin{array}{c}16.7 \\
(6 / 36)\end{array}$ & $\begin{array}{c}61.2 \\
(51.8)\end{array}$ \\
\hline & Regional & $\begin{array}{c}6.4 \\
(10 / 157)\end{array}$ & $\begin{array}{c}20.3 \\
(29.8)\end{array}$ \\
\hline \multirow[t]{2}{*}{$\begin{array}{l}\text { CCA6 Auctions } \\
\text { Losing bidders }\end{array}$} & Global & $\begin{array}{c}9.4 \\
(5 / 53)\end{array}$ & $\begin{array}{c}45.2 \\
(40.7)\end{array}$ \\
\hline & Regional & $\begin{array}{c}24.7 \\
(19 / 77)\end{array}$ & $\begin{array}{l}32.1 \\
(9.5)\end{array}$ \\
\hline \multirow[t]{2}{*}{ Winning bidders } & Global & $\begin{array}{c}9.8 \\
(5 / 51)\end{array}$ & $\begin{array}{c}52.4 \\
(16.4)\end{array}$ \\
\hline & Regional & $\begin{array}{c}9.9 \\
(13 / 131)\end{array}$ & $\begin{array}{l}23.7 \\
(7.7)\end{array}$ \\
\hline
\end{tabular}

\footnotetext{
${ }^{\mathrm{a}}$ Raw data in parentheses.

${ }^{\mathrm{b}}$ Standard error of the mean in parentheses.
}

bidders. These differences, which are statistically significant at the 1 percent level in a random-effects probit (controlling for repeated measures for the same subject), suggest a threshold problem; one, or both, of the regional bidders bids less aggressively, hoping that the other will cause prices to increase sufficiently to defeat the global bidder. The magnitude of the foregone profits was not that large averaging 14.9 and 32.1 ECUs in CCA4 and CCA6 auctions, respectively.

There are no comparable differences between global and regional bidders when they were winning with respect to foregone (potential) profits. This reinforces the notion of a threshold problem for losing bidders.

Our third observation is that bidders tend to bid much more often on their most profitable package than on their less profitable packages. Note, however, that the mere fact that the most profitable packages attract the most bids cannot prove that bidders are guided primarily by prices and profits. The same packages might be selected by other criteria. In many cases, particularly early on, the most profitable package and the "named" package coincide. The latter is the package of all items for the global bidder and the package of all positively valued items for the regional bidders. To establish the degree to which prices and profits guide bidding, we focus on those cases where named packages were different from the most profitable ones. Table 4 reports these data for the regional bidders. As shown, when there was a conflict between the named package and the most profitable package, and bidders chose to bid on only one of the two, the most profitable package attracts more attention from regional bidders in all rounds, often by a wide margin. Note, however, that the named package, or the named package and the most profitable package together, 
Table 4-Package Bids in CCA Auctions when Named Package No Longer the Most Profitable Bid ${ }^{\mathrm{a}}$

\begin{tabular}{lcccc}
\hline \hline & & \multicolumn{3}{c}{ Regional bidders } \\
\cline { 3 - 5 } & & $\begin{array}{c}\text { Percent } \\
\text { most profitable } \\
\text { only }\end{array}$ & $\begin{array}{c}\text { Percent } \\
\text { named package } \\
\text { only }\end{array}$ & $\begin{array}{c}\text { Percent both most } \\
\text { profitable and } \\
\text { named }\end{array}$ \\
\hline CCA4 Auctions & $(16)$ & 43.8 & 6.3 & 18.8 \\
Rounds 1-5 & $(126)$ & 42.1 & 9.5 & 14.3 \\
Rounds 6-10 & $(98)$ & 50.0 & 9.2 & 10.4 \\
Rounds 11-15 & $(35)$ & 40.0 & 11.4 & 5.7 \\
Rounds 16-20 & $(12)$ & 41.7 & 16.7 & 0.0 \\
Rounds > 20 & & & & \\
\hline CCA6 Auctions & $(7)$ & 57.1 & 0.0 & 14.3 \\
Rounds 1-5 & $(105)$ & 27.6 & 12.4 & 20.0 \\
Rounds 6-10 & $(92)$ & 26.1 & 9.8 & 10.9 \\
Rounds 11-15 & $(12)$ & 58.3 & 8.3 & 8.3 \\
Rounds 16-20 & $(9)$ & 33.3 & 22.2 & 11.1 \\
Rounds $>$ 20 & & & & \\
\hline
\end{tabular}

${ }^{a}$ Observations for which named package is not profitable are dropped. When a provisional winner does not bid, or the auction is in the last round, observations are dropped.

still attract a reasonable percentage of bids-which as shown below, has consequences for the actual data versus the simulation model.

Table 4 leaves out data for global bidders, as there were very few cases ( 2 for CCA4; 12 for CCA6) where the named package was not the most profitable package in a given round. Averaging over these few cases, only the most profitable package was bid on 42.8 percent of the time, with only the named package bid on 14.3 percent of the time, and both of them bid on 14.3 percent of the time - a pattern not unlike that reported for the regional bidders.

Finally, subjects typically did not place bids in rounds in which they were provisional winners. This effect was most pronounced in later rounds, when the auction had a greater chance of ending immediately. In auction rounds 11 and above, global (regional) bidders failed to submit new bids in 95.9 percent (89.2 percent) of all rounds in which they were provisional winners in CCA4 auctions, and in 90.6 percent ( 87.3 percent) for the CCA6 auctions. ${ }^{21}$ The reasons for these high frequencies are threefold: subjects do not bid in every round even when they are not provisional winners (see below); bidding on packages as a provisional winner can extend the auction and/or raise prices on provisionally winning bids with unknown consequences, so that provisional winners were willing to settle for what they already had; and given the bid patterns, more often than not the profit on the provisionally winning package was greater than or equal to the potential profit from any new package that could be bid on.

On this last point, in rounds 11 and higher, provisionally winning bidders rarely bid in a round in which their provisional profit was higher than they could earn on any new bid, with no new bids in 98.9 percent (95.5 percent) of all such cases for

\footnotetext{
${ }^{21}$ For rounds $1-10$, the corresponding percentages are 81.1 percent and 88.0 percent for global and regional bidders in CCA4 auctions and 63.6 percent and 71.1 percent for global and regional bidders in CCA6 auctions, respectively.
} 
Table 5-Outcomes in Easy versus Hard Package Auction Outcomes.

\begin{tabular}{|c|c|c|c|c|c|}
\hline & & \multicolumn{2}{|c|}{ CCA Efficiency } & \multicolumn{2}{|c|}{ SAA Efficiency } \\
\hline & & Average $^{b}$ & $\begin{array}{c}\text { Percent of } \\
\text { auctions } \\
\text { achieving } 100 \% \\
\text { efficiency }\end{array}$ & Average $^{b}$ & $\begin{array}{c}\text { Percent of } \\
\text { auctions } \\
\text { achieving 100\% } \\
\text { efficiency }\end{array}$ \\
\hline \multirow[t]{2}{*}{ 4-item auctions } & $\begin{array}{l}\text { Easy } \\
(9)^{\mathrm{a}}\end{array}$ & $\begin{array}{l}95.7 \% \\
(2.0)\end{array}$ & 88.9 & $\begin{array}{l}82.2 \% \\
(3.1)\end{array}$ & 50.0 \\
\hline & $\begin{array}{l}\text { Hard } \\
(8)^{\mathrm{a}}\end{array}$ & $\begin{array}{l}91.5 \% \\
(1.6)\end{array}$ & 35.4 & $\begin{array}{l}92.9 \% \\
(1.6)\end{array}$ & 50.0 \\
\hline \multirow[t]{2}{*}{ 6-item auctions } & $\begin{array}{c}\text { Easy } \\
(7)^{\mathrm{a}}\end{array}$ & $\begin{array}{l}95.3 \% \\
(1.8)\end{array}$ & 82.1 & $\begin{array}{l}83.9 \% \\
(2.0)\end{array}$ & 28.3 \\
\hline & $\begin{array}{l}\text { Hard } \\
(12)^{\mathrm{a}}\end{array}$ & $\begin{array}{l}92.4 \% \\
(1.2)\end{array}$ & 40.0 & $\begin{array}{l}90.9 \% \\
(1.1)\end{array}$ & 32.5 \\
\hline
\end{tabular}

${ }^{\text {a }}$ Number of value profiles.

${ }^{\mathrm{b}}$ Standard error of the mean in parentheses.

global (regional) bidders in the CCA4 auctions, and for 96.8 percent (88.7 percent) of all such cases for global (regional) bidders in the CCA6 auctions. Provisional winners stood pat somewhat less often when there was greater potential profit to be had on another package, with no new bids in 82.1 percent (57.9 percent) of all such cases for global (regional) bidders in the CCA4 auctions, and 61.8 percent (76.9 percent) of all cases for global (regional) bidders in the CCA6 auctions. Bidders were substantially more likely to bid following a round in which they had not secured a provisionally winning bid, bidding in 70.4 percent (60.7 percent) of all such cases in the CCA4 and in 75.7 percent (73.8 percent) of all cases for global (regional) bidders for CCA6 auctions. Finally, looking at those cases in which a provisionally winning bidder did not bid and was not winning on her most profitable package, the profit difference compared to their best alternative averaged 31.1 (13.2) ECUs for global (regional) bidders in the CCA4 auctions, and 54.7 (13.5) ECUs in the CCA6 auctions.

\section{B. Efficiency}

Efficiency is calculated as $\left(S_{\text {actual }}-S_{\text {random }}\right) /\left(S_{\max }-S_{\text {random }}\right)$, where $S_{\text {actual }}$ is the actual realized surplus from the auction, $S_{\text {random }}$ is the mean surplus resulting from a random allocation, and $S_{\max }$ is the maximum possible surplus. ${ }^{22}$ With this measure, in every environment, the mean efficiency of a random assignment of goods is 0 percent and the efficiency of a surplus-maximizing assignment is 100 percent.

Table 5 eports efficiency for CCA and SAA auctions with 4 and 6 items, categorizing the results into the previously defined easy (where the simulations achieve 100 percent efficiency) and hard cases (all other cases). Average efficiency is significantly higher in easy compared to hard CCA auctions, for both 4-item and 6-item

\footnotetext{
${ }^{22}$ The value of the random allocation is computed by taking the average of the surplus over all possible allocations $-3^{4}$ and $3^{6}$, respectively_assuming all items are sold in each auction.
} 
cases $(p<0.01) 23$ More dramatic yet are the differences in the frequency with which easy CCA auctions achieve 100 percent efficiency, averaging over 80 percent versus 40 percent or less for hard CCA auctions ( $p<0.01$ for both cases).

These efficiency differences between easy versus hard support hypothesis 2 , as the data summarized in the previous section are at least qualitatively consistent with the assumptions used in the simulations. Subjects bid on only a limited number of packages often including the currently most profitable ones. This suggests that the simulator may work more generally to distinguish hard versus easy environments for the CCA to achieve efficient outcomes.

As noted above, in our experiment, bidders do not rely solely on profits to decide which packages to bid on (recall Table 4). Bidders also bid on the named packages, even when they are not the most profitable ones. In the following we show how this behavior affects the efficiency of outcomes.

A closer look at auctions within the hard category show important and systematic differences. Although all the auctions in the hard category fail to achieve 100 percent efficiency in the simulations, for a number of these auctions the named packages correspond to the relevant packages, thereby attracting some attention even when they were not the most profitable packages ${ }^{24}$ In what follows, we will refer to these as "medium-hard" auctions. For the remaining auctions in the hard category, the relevant packages in terms of hypothesis 2 either involve all bidders getting one or more items, or splitting items between one of the regional bidders and the global bidder. This second subset of auctions, which we will refer to as "very hard," had lower average efficiency than the medium-hard auctions in the simulations. 25

Table 6 reports efficiencies for the medium-hard and very-hard auctions, as well as repeating the efficiency numbers for the easy auctions from Table 5. First, notice that average efficiency is substantially lower in the very-hard CCA auctions compared to either the easy or the medium-hard CCA auctions, as is the frequency with which these auctions achieve 100 percent efficiency ( $p<0.01$ in all cases). The medium-hard CCA auctions are much more comparable in terms of average efficiency to the easy CCA auctions than to the very hard CCA auctions. They achieve slightly higher average efficiency in the easy CCA4 auctions and slightly lower average efficiency in the easy CCA6 auctions. ${ }^{26}$ Medium-hard CCA auctions achieve 100 percent efficiency less often than the easy CCA auctions, with the difference significant at the 5 percent level for CCA6, and just missing statistical significance $(p=0.102)$ for CCA4 27

\footnotetext{
${ }^{23}$ Statistical tests comparing between CCA auctions in Tables 5, 6, and 8 are all one-tailed as they are based on simulated outcomes with clear predictions. Comparisons between CCA and SAA auctions in these tables are all two-tailed. Nonparametric Mann-Whitney tests continue to be used when conducting pair-wise tests for differences in average efficiency between cases. Binomial tests are used when conducting pair-wise tests for differences in frequencies of achieving 100 percent efficiency between cases.

${ }^{24}$ In the easy auctions all of the relevant packages are named packages as well as being the most profitable packages.

${ }^{25}$ For the 6-item case average efficiency for the very hard auctions was 90.6 percent in the simulations with only 1 of 6 auctions having average efficiency over 93 percent whereas efficiency averaged 95.1 percent for the mediumhard auctions with only one of 6 having efficiency lower than 93 percent. In the 4-item case efficiency averaged 87.5 percent for the very hard auctions versus 93.6 percent for the medium-hard case, with 2 (out of 6) of the very hard auctions having higher efficiency than the two medium-hard auctions.

${ }^{26}$ This difference is statistically significant at the 10 percent level using a one-tailed Mann-Whitney test for the CCA6 auctions. But not significant at conventional levels for the CCA4 case.

${ }^{27}$ One-tailed binomial tests in both cases.
} 
Table 6-Outcomes in Medium Hard versus Very Hard Package Auction Outcomes

\begin{tabular}{|c|c|c|c|c|}
\hline & \multicolumn{2}{|c|}{ CCA Efficiency } & \multicolumn{2}{|c|}{ SAA Efficiency } \\
\hline & Average $^{\mathrm{b}}$ & $\begin{array}{c}\text { Percent of } \\
\text { auctions } \\
\text { achieving } 100 \% \\
\text { efficiency }\end{array}$ & Average $^{b}$ & $\begin{array}{c}\text { Percent of } \\
\text { auctions } \\
\text { achieving 100\% } \\
\text { efficiency }\end{array}$ \\
\hline \multicolumn{5}{|l|}{ 4-item auctions } \\
\hline Easy $(9)^{\mathrm{a}}$ & $\begin{array}{l}95.7 \% \\
(2.0)\end{array}$ & 88.9 & $\begin{array}{l}82.2 \% \\
(3.1)\end{array}$ & 50.0 \\
\hline Medium hard $(2)^{\mathrm{a}}$ & $\begin{array}{l}96.4 \% \\
(2.2)\end{array}$ & 75.0 & $\begin{array}{l}92.6 \% \\
(2.1)\end{array}$ & 25.0 \\
\hline Very hard $(6)^{a}$ & $\begin{array}{l}89.9 \% \\
(1.9)\end{array}$ & 22.2 & $\begin{array}{l}93.0 \% \\
(2.0)\end{array}$ & 58.3 \\
\hline \multicolumn{5}{|l|}{ 6-item auctions } \\
\hline $\operatorname{Easy}(7)^{\mathrm{a}}$ & $\begin{array}{l}95.3 \% \\
(1.8)\end{array}$ & 82.1 & $\begin{array}{l}83.9 \% \\
(2.0)\end{array}$ & 28.3 \\
\hline Medium hard (6) ${ }^{\mathrm{a}}$ & $\begin{array}{l}94.2 \% \\
(1.6)\end{array}$ & 59.4 & $\begin{array}{l}87.8 \% \\
(1.3)\end{array}$ & 15.8 \\
\hline Very hard $(6)^{a}$ & $\begin{array}{l}90.7 \% \\
(1.7)\end{array}$ & 21.2 & $\begin{array}{l}93.8 \% \\
(1.5)\end{array}$ & 48.7 \\
\hline
\end{tabular}

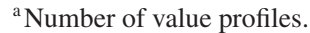

${ }^{\mathrm{b}}$ Standard error of the mean in parentheses.
}

Efficiency in the CCA auctions is not uniformly higher than in the SAA auctions. In the easy category, the CCA auctions yield significantly higher efficiency than the corresponding SAA auctions $(p<0.01)$, averaging a little over 10 percent higher efficiency, and the easy CCA auctions achieve 100 percent efficiency substantially more often than in the corresponding SAA auctions $(p<0.01)$. But in the hard category, average efficiency in the CCA auctions is not significantly different from the SAA auctions $(p>0.20)$. For very-hard cases, the CCA had lower average efficiency, and substantially lower frequencies of 100 percent efficiency, than the corresponding SAA auctions for both the 4 - and 6-item auctions ( $p<0.05$ in all cases).

These findings provide evidence that the relative efficiency of the CCA and SAA auction mechanisms depend on the parameter configurations. Further, the similarity in outcomes between the easy and medium-hard CCA auctions serve to contradict any hypothesis that behavior in our experiment is entirely guided by price and profits (as it is in our simulation). Rather, the data indicate that there is little difference in outcome efficiency between the easy and medium-hard cases, suggesting that named package play an important role in guiding bidding. Very hard auctions provide further evidence consistent with this explanation, as bidding on the single most profitable package or the named package does not consistently point bidders to the efficient outcome in these cases. This is a reminder, if any is needed, about the limits of simulators and the importance of identifying general properties of actual bidder behavior when attempting to predict auction outcomes.

\section{Seller Revenue}

For a competitive-revenue standard for package auctions, we follow Milgrom (2007) in using the minimum seller revenue at any core allocation. The core for 
TABle 7-Revenue and Profits in CCA and SAA Auctions

(Standard error of the mean in parentheses)

\begin{tabular}{|c|c|c|c|c|c|c|c|c|}
\hline & \multicolumn{2}{|c|}{ Revenue $^{\mathrm{b}}$} & \multicolumn{2}{|c|}{ Profit $^{\mathrm{c}}$} & \multicolumn{2}{|c|}{ Global profit $^{\mathrm{c}}$} & \multicolumn{2}{|c|}{ Local profit ${ }^{\mathrm{c}}$} \\
\hline & $\mathrm{CCA}$ & SAA & $\mathrm{CCA}$ & SAA & $\mathrm{CCA}$ & SAA & $\mathrm{CCA}$ & SAA \\
\hline \multicolumn{9}{|c|}{ 4-item auctions } \\
\hline Easy $^{\mathrm{a}}(9)$ & $\begin{array}{l}92.1 \% \\
(2.0)\end{array}$ & $\begin{array}{l}98.6 \% \\
(2.4)\end{array}$ & $\begin{array}{l}21.4 \% \\
(1.2)\end{array}$ & $\begin{array}{l}8.8 \% \\
(2.7)\end{array}$ & $\begin{array}{l}5.1 \% \\
(1.2)\end{array}$ & $\begin{array}{c}-3.4 \% \\
(1.5)\end{array}$ & $\begin{array}{l}8.2 \% \\
(0.8)\end{array}$ & $\begin{array}{l}6.1 \% \\
(0.9)\end{array}$ \\
\hline $\operatorname{Hard}^{\mathrm{a}}(8)$ & $\begin{array}{l}92.9 \% \\
(2.4)\end{array}$ & $\begin{array}{c}102.2 \% \\
(2.7)\end{array}$ & $\begin{array}{l}20.1 \% \\
(1.8)\end{array}$ & $\begin{array}{l}12.4 \% \\
(2.7)\end{array}$ & $\begin{array}{l}3.4 \% \\
(0.9)\end{array}$ & $\begin{array}{c}-2.6 \% \\
(1.4)\end{array}$ & $\begin{array}{l}8.4 \% \\
(0.9)\end{array}$ & $\begin{array}{l}7.5 \% \\
(1.0)\end{array}$ \\
\hline \multicolumn{9}{|c|}{ 6-item auctions } \\
\hline Easy $^{\mathrm{a}}(7)$ & $\begin{array}{l}90.4 \% \\
(3.1)\end{array}$ & $\begin{array}{l}94.2 \% \\
(3.0)\end{array}$ & $\begin{array}{l}18.5 \% \\
(2.4)\end{array}$ & $\begin{array}{l}8.7 \% \\
(2.6)\end{array}$ & $\begin{array}{l}4.6 \% \\
(2.1)\end{array}$ & $\begin{array}{c}-0.8 \% \\
(1.4)\end{array}$ & $\begin{array}{l}6.9 \% \\
(1.0)\end{array}$ & $\begin{array}{l}4.7 \% \\
(1.0)\end{array}$ \\
\hline $\operatorname{Hard}^{\mathrm{a}}(12)$ & $\begin{array}{l}92.9 \% \\
(1.4)\end{array}$ & $\begin{array}{l}86.7 \% \\
(2.5)\end{array}$ & $\begin{array}{l}11.9 \% \\
(1.2)\end{array}$ & $\begin{array}{l}16.2 \% \\
(2.3)\end{array}$ & $\begin{array}{l}4.2 \% \\
(1.0)\end{array}$ & $\begin{array}{l}4.5 \% \\
(1.1)\end{array}$ & $\begin{array}{l}3.8 \% \\
(0.5)\end{array}$ & $\begin{array}{l}5.9 \% \\
(0.9)\end{array}$ \\
\hline
\end{tabular}

\footnotetext{
${ }^{\text {a }}$ Easy versus Hard categories defined as in Table 5 above. Number of value profiles in parentheses.

${ }^{\mathrm{b}}$ Measured as a percentage of minimum revenue in the core.

${ }^{\mathrm{c}}$ Measures as a percentage of the efficient allocation.
}

package allocation problems has a competitive auction interpretation: an individually rational allocation is in the core if there is no group of bidders who could all do better for themselves and for the seller by raising some of their losing bids. To enhance comparability, we report revenue in each auction as a percentage of the minimum revenue in the core. The experimental outcomes are reported in Table 7.

Our main hypotheses do not concern levels of revenues and profits. Compared to efficiencies, which are about allocations, revenue and profits may depend more sensitively on bidder behavior and the naïve bidding behavior used in the simulation may not be sufficiently descriptive to predict these outcomes. Also, the parameter selection procedure was based on outcome efficiencies, which is not the most suitable choice when evaluating how environments affect revenues and profits. We nevertheless report outcomes based on the hard and easy cases.

We find no evidence that the revenues in the CCA differ significantly between the hard and easy categories $(p>0.50) .28$ Thus, the factors that lead to high efficiencies may differ from those that lead to high revenues in CCA. Comparing CCA with SAA, the only significant difference identified is that revenue in the SAA4 hard auctions is significantly higher $(p<0.01)$ than in the CCA4 hard auctions. Note that in this one case, average revenue in the SAA auctions is over 100 percent of minimum revenue in the core. We will return to this point in the next section where profits are discussed.

There are no directly equivalent revenue results from other multi-unit auction experiments. Porter et al. (2003) do not report revenue comparisons between auction mechanisms. Brunner et al.'s (2007) normalization reports revenue as a percentage of the efficient allocation. ${ }^{29}$ They find that revenue is significantly higher in their version of the CCA auction than the simultaneous multi-round (SMR) auction employed by

\footnotetext{
${ }^{28}$ Statistical tests for Table 7 are all two-tailed, Mann-Whitney tests using each auction as the unit of observation.

${ }^{29}$ Brunner et al. (2007) use actual revenue less the revenue from a random allocation, in which bidders pay full value, in the numerator, and revenue from the efficient allocation less the revenue from a random allocation in the denominator, so that the difference lies in taking differences from average revenue resulting from a random allocation in both the numerator and denominator.
} 
the FCC (the closest relative to our SAA auction). This contrasts with our results as revenues are not significantly different in the six-item case and are significantly higher in the SAA in the four-item hard case. Their auctions involve bidding over more items than ours and include two global bidders competing over the same set of licenses. Also, because their mechanism withholds information about provisionally winning bids, it may encourage more bidding. Their revenue results hold for both high- and low-synergy cases. However, this comparison is strained by the fact that they had a relatively large number of items left unsold in their SMR auctions.

\section{Bidder Profits}

Table 7 reports profits as a percentage of the efficient allocation. As between hard and easy CCA auctions, total profit is significantly higher in the easy CCA6 auctions $(p<0.01)$, with most of the difference being accounted for by the higher profits for regional bidders in the easy auctions $(p<0.01)$. Comparing between CCA and SAA auctions, total profits are markedly higher in the CCA auctions for the easy and hard 4 -item auctions as well as the easy 6 -item auctions ( $p<0.05$ in all cases). For the remaining hard 6-item case there are no significant differences between CCA and SAA auctions, with total profits somewhat higher in the SSA auctions for the hard case (but $p>0.10$ ). Note that in each of the three cases where the CCA auctions generate higher profits, global bidders earn negative average profits in the SAA auctions, while the regional bidders earn positive average profits. These negative profits are reflective of the exposure problem which is eliminated in the CCA auctions and which is more severe for global as opposed to regional bidders within the SAA auctions. Further, it is these losses that contribute to revenue exceeding 100 percent of minimum revenue in the core for hard auctions in the SAA4 auctions mentioned earlier.

Our profit results stand in marked contrast to those reported in Brunner et al. (2007), where total bidder profits are lower, sometimes substantially lower, in the CCA compared to the SMR auctions. Differently from Brunner et al. (2007), we announced provisional winners following each round of bidding. Failure to reveal provisional winners may cause bidders to raise their own winning bids at least some of the time. In our design bidders who do not raise their own provisionally winning bids could still "bid against themselves" by placing a bid that overlaps with their provisionally winning bid. However, this is unlikely to occur in later auction rounds when final prices are set.

\section{E. Distance from the Core}

Table 8 reports distances from the core for both experimental outcomes and simulated auction outcomes, using a revised definition of easy versus hard. Our original definitions of easy versus hard were based on evaluating the efficiency of simulation outcomes, and easy cases for efficiency may not also be ones in which the simulated behavior lies close to the core. Nevertheless, to the extent that the simulator reflects actual behavior, it may also be helpful in making predictions about the distance of outcomes from the core. We use the simulator to create another classification based in part on distance from the core. 
Table 8-Simulated Outcomes versus Experimental Outcomes: Distance From the Core

\begin{tabular}{|c|c|c|c|c|c|}
\hline & & \multicolumn{2}{|c|}{ Auction outcomes ${ }^{b}$} & \multicolumn{2}{|c|}{ Simulation outcomes $^{\mathrm{c}}$} \\
\hline & & $\begin{array}{l}\text { Average } \\
\text { distance }\end{array}$ & $\begin{array}{c}\text { Percent of } \\
\text { auctions } \\
\text { achieving zero } \\
\text { distance }\end{array}$ & $\begin{array}{l}\text { Average } \\
\text { distance }\end{array}$ & $\begin{array}{c}\text { Percent of } \\
\text { auctions } \\
\text { achieving zero } \\
\text { distance }\end{array}$ \\
\hline \multirow[t]{2}{*}{ 4-item auctions } & $\begin{array}{l}\text { Core-easy } \\
(8)^{\mathrm{a}}\end{array}$ & $\begin{array}{l}14.0 \% \\
(3.3)\end{array}$ & 18.8 & $\begin{array}{l}4.6 \% \\
(5.0)\end{array}$ & 44.3 \\
\hline & $\begin{array}{c}\text { Core-hard } \\
(9)^{\mathrm{a}}\end{array}$ & $\begin{array}{l}21.9 \% \\
(2.4)\end{array}$ & 3.7 & $\begin{array}{l}29.7 \% \\
(20.4)\end{array}$ & 5.3 \\
\hline \multirow[t]{2}{*}{ 6-item auctions } & $\begin{array}{c}\text { Core-easy } \\
(5)^{\mathrm{a}}\end{array}$ & $\begin{array}{l}18.6 \% \\
(4.9)\end{array}$ & 10.7 & $\begin{array}{l}5.0 \% \\
(5.1)\end{array}$ & 39.7 \\
\hline & $\begin{array}{c}\text { Core-hard } \\
(14)^{\mathrm{a}}\end{array}$ & $\begin{array}{l}20.5 \% \\
(2.2)\end{array}$ & 2.6 & $\begin{array}{l}24.9 \% \\
(14.9)\end{array}$ & 1.8 \\
\hline
\end{tabular}

${ }^{\mathrm{a}}$ Number of value profiles.

${ }^{\mathrm{b}}$ Standard error of the mean in parentheses.

${ }^{\mathrm{c}}$ Standard error in parentheses.

Auctions are categorized as core-easy if, in the simulations, they achieve 100 percent efficiency and the scaled distance from the core is no greater than 15 percent ${ }^{30}$ All other auctions, whether they achieve 100 percent efficiency or not, are classified as core-hard. Raw distance from the core is defined as the maximum violation of one of the inequalities defining the core. The scaled distance is the raw distance divided by the difference between full efficiency and efficiency resulting from randomly allocating items among bidders.

For the simulated outcomes, the average scaled distance from the core is 5 percent or less for the core-easy CCA auctions versus 24 percent or more for the core-hard ones, with corresponding differences in the fraction of simulated outcomes achieving zero distance from the core. The experimental outcomes do not achieve the same magnitude of difference between core-easy and core-hard as the simulations, but the differences are in the right direction for both the four- and six-item cases. This difference is statistically significant at the one percent level in the CCA4 auctions. The difference between core-easy and core-hard is not as large in the CCA6 auctions, with average distance from the core significant at the 10 percent level, and percent of auctions with 0 distance from the core significant at the 5 percent level.

Although our categorization of core-easy versus core-hard in terms of the simulations qualitatively predicts the experimental outcomes, it does not predict the levels accurately. Incorporating more details of bidder behavior into the simulation could narrow the discrepancy. But fine-tuning the bidder behavior model is not the main purpose of this paper.

\footnotetext{
${ }^{30}$ Results are robust to alternative definitions, such as the simulated distances from the core being no greater than 10 percent. None of the auctions achieving 100 percent efficiency also achieve 0 distance from the core. Employing a criterion of simulated distance from the core being no greater than 5 percent yields 2 CCA4 core-easy auctions and 1 CCA6 core-easy auction, too small a sample for meaningful results.
} 


\section{F. Rounds to Auction Completion}

The average number of rounds to completion was quite similar across auction mechanisms. For the auctions in the easy category, CCA6 (CCA4) auctions required an average of 15.5 (16.7) rounds per auction versus 18.5 (15.4) rounds for the SAA6 (SAA4) auctions. For the hard category, CCA6 (CCA4) auctions required an average of 17.5 (17.8) rounds per auction versus 18.0 (15.2) rounds for the SAA6 (SAA4) auctions. None of these differences is statistically significant at conventional levels.

The one thing that does stand out in the data is that, not surprisingly, total bidder profits decrease systematically as the number of rounds in a given auction increase, regardless of which auction mechanism is used. For example, average profits of provisionally winning bidders decreased monotonically over rounds $1-5,6-10,11-15$, $15-20$ and 20 or greater in the CCA6 auctions, going from a high of 208.4 ECUs in rounds $1-5$ to a low of 17.6 ECUs in rounds greater than 20 for global bidders, and from a high of 113.6 ECUs to a low of 22.9 ECUs for regional bidders.

\section{Conclusion}

We employ theory and experiment to investigate when "standard" package auctions - and particularly the combinatorial clock auction (CCA) - lead to efficient or, more strongly, core outcomes in package allocation problems. Our analysis begins with two theorems asserting that if winning bidders bid most aggressively on their efficiency-relevant or core-relevant packages, and if losing bidders exhaust their profit opportunities, then the result is an efficient or core outcome, respectively. Our experiment uses the CCA and a matched simultaneous ascending auction (SAA).

In principle, differences in performance among auction environments could depend on how many packages subjects bid on in different situations, so we begin by looking for that. We find no evidence that these numbers vary between the easy and hard auction environments. Instead, bidders in our experiment typically bid on just the one or two most profitable packages and those packages often remained unchanged for many rounds during an auction. In our data, consistent with our theory, the CCA yields efficient allocations and core-level revenues most frequently when the packages that are selected by this sort of behavior are the relevant ones. We verify this by comparing the outcomes of our experiment to those of simple simulations, in which automated bidders bid only for the single most profitable package at each round.

The outcomes from our experiment are largely consistent with the two hypotheses we formulated in Section I: Subjects bid for many fewer packages than the profitable ones at the current round prices, and simulations based on such bidder behavior qualitatively help predict the efficiency of outcomes. Simulations were less effective, however, at predicting the distance of experimental outcomes from the core.

Our finding, that price-guided auctions can fail to direct bidders to relevant packages early enough in the auction, suggests possible improvements to the auction design. This failure could be greater in environments in which, unlike most of our auctions, bidders may have many core-relevant packages. One possible refinement is to make relevant bids more likely by making it easier to bid on more sets of 
licenses. That might be accomplished by implementing a richer bidding language than the XOR language of our experiment.

A second element in our CCA design inhibiting bidding on the relevant packages is the reporting of provisionally winning bids. This impacts outcomes in two ways: by reporting provisionally winning bids may help bidders to tacitly collude, stopping bidding early on if all bidders are satisfied with their current profits, and reporting provisionally winning bids encourages bidders to adopt a wait-and-see policy, hoping that a provisionally winning bid with a large profit margin will eventually become a winner-particularly in the later rounds of the auction. There was at least one clear case of tacit collusion with bidding ending in round 3, with prices at their starting values and substantial profits for all bidders ${ }^{31}$ One way to control for such implicit collusion is to employ a tie-breaking rule which allocates packages to the smallest possible number of bidders, instead of randomly as in our experimental design. This would maximize the number of bidders without provisionally winning bids in early rounds thereby promoting defection from such tacit collusion. The provisionally winning bidders' wait-and-see policy can generate inefficient assignments and/or noncore (low revenue) outcomes. This happens because such bidders may fail to bid on alternative packages with greater potential profits and may miss opportunities to place higher bids on the same package. Withholding information about provisionally winning bids would eliminate the wait-and-see motive, but would add other difficult strategic choices.

As emphasized in the introduction, the set of package auction environments is far too large to be convincingly explored with experiments alone. The simulation approach provides a predictive theory that offers the promise of generalizable conclusions. In our experiment, simulations, which make specific predictions about efficiency, revenue, and the core, had some predictive success. We believe that simulations are a promising tool for testing and designing practical auction mechanisms.

\section{REFERENCES}

Ausubel, Lawrence M., Peter Cramton, and Paul Milgrom. 2005. "The Clock-Proxy Auction: A Practical Combinatorial Auction Design.” In Combinatorial Auctions, ed. Peter Cramton, Yoav Shoham, and Richard Steinberg, 115-38. Cambridge, MA: MIT Press.

Ausubel, Lawrence M., and Paul R. Milgrom. 2002. "Ascending Auctions with Package Bidding." B. E. Journal of Theoretical Economics: Frontiers of Theoretical Economics, 1(1).

Bernheim, B. Douglas, and Michael D. Whinston. 1986. "Menu Auctions, Resource Allocation, and Economic Influence.” Quarterly Journal of Economics, 101(1): 1-31.

Blumrosen, Liad, and Noam Nisan. 2005. "On the Computational Power of Iterative Auctions." ACM Conference on Electronic Commerce (EC'05), Vancouver, Canada.

-Brunner, Christoph, Jacob K. Goeree, Charles A. Holt, and John O. Ledyard. 2010. “An Experimental Test of Flexible Combinatorial Spectrum Auction Formats." American Economic Journal: Microeconomics, 2(1): 39-57.

Day, Robert, and Paul Milgrom. 2007. "Core-Selecting Package Auctions." International Journal of Game Theory, 36(3-4): 393-407.

\footnotetext{
${ }^{31}$ In this case, no new bids were placed after round 1. This happened midway through the second full session, so that subjects would have correctly anticipated that they could not do much better by continuing to compete. Profits were 45, 26, and 43 ECUs for the 2 regional bidders and the global bidder, respectively.
} 
Goeree, Jacob K., Charles A. Holt, and John O. Ledyard. 2007. "An Experimental Comparison of Flexible and Tiered Package Bidding." Report to the Wireless Telecommunications Bureau of the Federal Communications Commission. May 25, 2007.

-Gul, Faruk, and Ennio Stacchetti. 1999. "Walrasian Equilibrium with Gross Substitutes." Journal of Economic Theory, 87(1): 95-124.

Hayek, Friedrich A. 1945. "The Use of Knowledge in Society." American Economic Review, 35(4): 519-30.

Hurwicz, Leonid. 1977. "On the Dimensional Requirements of Informationally Decentralized ParetoSatisfactory Processes." In Studies in Resource Allocation Processes, ed. Kenneth J. Arrow and Leonid Hurwicz, 413-24. New York: Cambridge University Press.

Kwasnica, Anthony M., John O. Ledyard, David Porter, and Christine DeMartini. 2005. "A New and Improved Design for Multi-Object Iterative Auctions." Management Science, 51(3): 419-34.

- Milgrom, Paul. 2000. "Putting Auction Theory to Work: The Simultaneous Ascending Auction." Journal of Political Economy, 108(2): 245-72.

Milgrom, Paul. 2007. "Package Auctions and Exchanges." Econometrica, 75(4): 935-65.

Nisan, Noam, and Ilya Segal. 2006. "The Communication Requirements of Efficient Allocations and Supporting Prices." Journal of Economic Theory, 129(1): 192-224.

Porter, David, Stephen Rassenti, Anil Roopnarine, and Vernon Smith. 2003. "Combinatorial Auction Design." Proceedings of the National Academy of Sciences, 100(19): 11153-57. 


\section{This article has been cited by:}

1. Ali Hortaçsu, David McAdams. 2018. Empirical Work on Auctions of Multiple Objects. Journal of Economic Literature 56:1, 157-184. [Abstract] [View PDF article] [PDF with links]

2. Pallab Sanyal. 2016. Characteristics and Economic Consequences of Jump Bids in Combinatorial Auctions. Information Systems Research 27:2, 347-364. [Crossref]

3. Simon Loertscher, Leslie M. Marx, Tom Wilkening. 2015. A Long Way Coming: Designing Centralized Markets with Privately Informed Buyers and Sellers. Journal of Economic Literature 53:4, 857-897. [Abstract] [View PDF article] [PDF with links]

4. Jeroen Hinloopen, Sander Onderstal. 2014. Going once, going twice, reported! Cartel activity and the effectiveness of antitrust policies in experimental auctions. European Economic Review 70, 317-336. [Crossref]

5. John H. Kagel, Yuanchuan Lien, Paul Milgrom. 2014. Ascending prices and package bidding: Further experimental analysis. Games and Economic Behavior 85, 210-231. [Crossref]

6. Xiaoqiu Qiu, Dirk Neumann. Accelerating Combinatorial Clock Auctions Using Bid Ranges 231-240. [Crossref]

7. Anthony M. Kwasnica, Katerina Sherstyuk. Multiunit Auctions 75-108. [Crossref]

8. M S Iftekhar, A Hailu, R K Lindner. 2013. Choice of item pricing feedback schemes for multiple unit reverse combinatorial auctions. Journal of the Operational Research Society 64:11, 1571-1582. [Crossref]

9. Anthony M. Kwasnica, Katerina Sherstyuk. 2013. MULTIUNIT AUCTIONS. Journal of Economic Surveys 27:3, 461-490. [Crossref]

10. Martin Bichler, Pasha Shabalin, Georg Ziegler. 2013. Efficiency with Linear Prices? A GameTheoretical and Computational Analysis of the Combinatorial Clock Auction. Information Systems Research 24:2, 394-417. [Crossref]

11. Peter Cramton. 2013. Spectrum Auction Design. Review of Industrial Organization 42:2, 161-190. [Crossref]

12. J.G. Tisdell, Md Sayed Iftekhar. 2013. Fisheries quota allocation: Laboratory experiments on simultaneous and combinatorial auctions. Marine Policy 38, 228-234. [Crossref]

13. Tobias Scheffel, Georg Ziegler, Martin Bichler. 2012. On the impact of package selection in combinatorial auctions: an experimental study in the context of spectrum auction design. Experimental Economics 15:4, 667-692. [Crossref]

14. Kirill Chernomaz, Dan Levin. 2012. Efficiency and synergy in a multi-unit auction with and without package bidding: An experimental study. Games and Economic Bebavior 76:2, 611-635. [Crossref]

15. Gediminas Adomavicius, Alok Gupta, Pallab Sanyal. 2012. Effect of Information Feedback on the Outcomes and Dynamics of Multisourcing Multiattribute Procurement Auctions. Journal of Management Information Systems 28:4, 199-230. [Crossref]

16. Gediminas Adomavicius, Shawn P. Curley, Alok Gupta, Pallab Sanyal. 2012. Effect of Information Feedback on Bidder Behavior in Continuous Combinatorial Auctions. Management Science 58:4, 811-830. [Crossref]

17. Ryuji Sano. 2011. Non-bidding equilibrium in an ascending core-selecting auction. Games and Economic Behavior. [Crossref] 